\title{
Application Research on Agricultural Production Throughout the Internet of Things
}

\author{
Peng Qin, Zhao Lu and Tao Zhu
}

Nanchang Key Laboratory of material and structure detection Jiangxi University of Technology

Keywords: Internet of things; Agricultural product production; RFID; Application

\begin{abstract}
The Internet of things is a kind of network connecting any things to the Internet based on the existing RFID system of the Internet and other information sensing devices according to the predetermined protocol for information exchange and communication so as to achieve intelligent identification, positioning, monitoring, tracking and remote control. It has become the expanded and extended network on the basis of the Internet. The application of the Internet of things in agricultural product production and research accelerates the process of agricultural modernization, and guarantees the security of agriculture as the basic industry. For a long time, harvest of agricultural production is based on the weather, so it is hardly to dominate the arrangement of various activities according in agricultural production with scientific means. With the popularity of IPv6 technology as well as the full use of the ZigBee technology in the low-power and short-range environment, it is believed that with the increasing popularity of the Internet of things in agricultural production, more and more agricultural producers will benefit from it.
\end{abstract}

\section{Introduction}

Agriculture is a basic industry related to people's livelihood, so it is of vital importance to make it be full of information and become intelligent. The application of the Internet of things in agricultural production and scientific researches is of great significance to establish intensive agricultural production, improve disease prevention of plants and animals as well as agricultural products' quality and security. Full monitoring of agricultural breeding, growing, harvesting, transportation and sales enables people to check the source of agricultural products, so it is a leaping step of modern agriculture based on the new informationized application, and the Internet of things is a new technological revolution because it make a qualitative leap in agricultural production, and these new functions will further make agricultural production be efficient, convenient and safe so as to form new agriculture based on this function.

\section{Analysis of the Demand for Internet of Things in Agricultural Production}

Role of Internet of Things in the Increase of Agricultural Production. The National Agricultural Information Engineering Technology Research Center is dedicated in the application and research of information technologies in agriculture, vigorously promoting the development of agricultural information in China. By installing ecological information by wireless sensors and other intelligent control systems in agricultural production areas, they test ecological environment of the 
entire production area so as to timely master some parameters affecting environment of the production areas, and timely regulate the irrigation system and insulation system according to changes of parameters and then ensure the best environment for the growth of crops so as to increase production and guarantee the quality of crops.

Role of Internet of Things in Agricultural Products Logistics and Quality Security. Due to the frequent occurrence of issues about the security of agricultural products, the security of agricultural products is receiving more and more attention with monitor on each production link of the agricultural products, such as planting, cultivating, growing, flowering, fruiting and harvesting, enable important phrases like growth environment and drug fertilization during the entire process of producing agricultural products to get intelligent documented and well-based. The use of RFID frequency technology and two-dimensional code to record data of each stage can make customers check various information through the terminal device or the Internet when they get the agricultural products, and then they can feel assured to eat these products.

\section{Application of Internet of Things in Agricultural Production}

Application of Internet of Things in Agricultural Plant Production. Internet of things technologies in crop growth monitoring can be achieved in irrigation, soil air monitoring of changes, as well as a large area of the earth's surface, temperature, humidity, rainfall, wind, atmosphere and land moisture, nitrogen concentration, and soil $\mathrm{pH}$ values and other information, data is transmitted over a mobile communications network for service management platform, Service management platform is for data analysis and processing. To scientific predictions, take steps to help farmers, disaster mitigation, scientific planting and improve the overall efficiency of agriculture. A range of crops grown in different periods had been "ambiguous" issues addressed agriculture IOT are real-time quantitative "exact" checks, just flick a switch, and listen to IOT "instruction", which will be able to grow a variety of vegetables. The following is the three-layer structure adopted by intelligent planting system solution proposed by $\mathrm{Da}$ Tang, and it is made up of sensing layer, transmitting layer and application layer.

Application of Internet of Things in Agricultural Cultivation Production. With the rapid development of the aquaculture ind ustry and the continuous improvement of large-scale, specialized, transform, upgrade the traditional modern aquaculture cultivation, innovation, speed up the modernization of farming, information technology have become an increasingly important factor in aquaculture development. New aquaculture animals networked integrated information solutions using bar code labels (barcode, QR code) and identification technologies such as RFID tags, smart technology, large database and WEB technology for mobile terminals as well as Android application development technology, and many other mobile Internet technology and Internet of things technologies, make full use of modern information technology in modern animal husbandry management and services. New aquaculture moved animal networking integrated information solution program from agricultural farming, acquisition, processing, transport, sales and all links of identifies, recognition, track and query, warehouse, assets and enterprise information and all related management, which has achieved aquaculture focus core business of full information, business management information, management information resources and information service standardized. Mass of modernization farming providing with important information, support and service makes aquaculture of information level follow real networking of quickly development. Production management system based on the Internet key technology applications, using bar code technology 
and RFID technology to identify the animals and equipment, related recognizer for mobile intelligent terminal with integrated production management through the Android application. And moving through the terminal sends the data to back-end systems, in order to achieve the production of mobile office.

\section{Difficulties in the Application of Internet of Things in Agricultural Production and Solutions}

Difficulties in the Development of Internet of Things. Address Problem. In agricultural production, due to a large expansion of vegetables and thousands of animals, each type of bottom sensor is widely distributed. However, various sensor only needs network marked to and upper gateway links, currently existing of network marked IPv4 has depletion, led no enough of network address to dominated to bottom sensor, many of objects need each distribution to have only of marked and upper gateway links, existing IP address with close depletion, used private of address and needs independent of address parsing system, which has increased the server of burden.

Standard Problem. The rapid development of the Internet should be attributed to good standardization of problem-solving, global agreement on Protocol for transmitting TCP/IP protocols, routers, Terminal architecture and operating system. In the development of the Internet of things, sensor and application at all levels will have a lot of technology, needed to unify technical standard. Forming a management mechanism for the Internet of things will bring to our lives more comfortable, convenient and safe time.

Price Proble m. Real networking in commercial application aspects business mode also is not clear, in addition, current production real networking by needed of chip, component of costs also compares high. Currently, it cost about 1.5 yuan to make a label. High cost decided the technology of this item currently can only be used in value-added relative place with high merchandise, putting all items implanted recognition chip. This point now seems also not too reality, which greatly limits the range of promotion of RFID application .

Technical Platform Problem. Internet of things is made up of sensor network, RFID reading device, barcode and the Internet.

Intelligent Processing Problem. Intelligent signal processing will make necessary process to various original data obtained by the collection equipment so as to obtain information related to the targeted objects. Signal feature extraction can be made in the mapping space, using signal analysis techniques, such as characteristics, classification techniques, and various features, which can signal corresponding to a certain type of physical events.

Security Proble m. Because of the private property of the end sensor network of Internet of things, the problem of security must be faced. Sensor nodes in the Internet of things usually require the deployment of unmanned, uncontrollable environment, in addition to Wi-Fi information disclosure, information tampering and replay attacks, denial of service, and many other threats, which are easy for an attacker to obtain, through physical means to obtain all the information stored in the node, which penetrated the network, network threats.

\section{Solutions to the Application and Popularity of Internet of Things in Agricultural Production}

Technical Solutions. IPv6 is the Internet Protocol Version 6, the initials, IPv6 is the IETF (Internet Engineering Task Force), designed to replace the existing version of the IP Protocol (IPv4), next-generation IP protocol. Pv6 uses a 128-bit address length, has very amazing address resources, 
IPv6 can give the address of every grain of dust in the universe, its promotion will create the conditions for all things connected, will also be the basis of networking technology. Mass of address resources support number to trillion meter of items, interconnected of premise is first needs network address to positioning these items, face mass of address distribution problem, past of manual distribution and DHCP technology has unable to dominate address, no state address distribution is reasonable solution the problem of method, through set simple of distribution process, node obtained has network address and interface address, achieved has Plug-and-play of concept. IPv6 was designed taking into account features of the object at the beginning of the related design, met in agricultural production, animal husbandry, and fishing the mobility characteristics of the object.

ZigBee is a low-power local area network protocol IEEE802.15.4 standard. It is s a short-range, low-power wireless communication technology. The origin thought of the technology is to have developed from the bees. Characterized by a closer, self-organizing, low power, high rate information transmission, ZigBee is a cheap, low-power short-range wireless network communications technology. ZigBee is a new type of wireless network technology with short distance, low rate and low energy consumption while featured by low complexity, low power, low rate, low costs, establishing network on its own and reliability, etc. and it is mainly applied to automatic control and far-distance control, etc. It is because of this special feature and ZigBee's application in agricultural production that various intense sensors are needed in agricultural production. However, due to high costs, most sensors cannot use high-frequency processors and sensor equipment with high capacity to a wide range, and the popularity of ZigBee technology enables that any equipment can be embedded into low-power sensors.

Non-technical Solutions. Popularity of the Internet of things is not only limited to the development of technologies. First of all, the popularity of the Internet is not like consuming ordinary electronic products, the explicit demands of which are easily met with a large amount of both demands and supplies. Therefore, its growth curve is always very steep. Users of Internet of things are not only the general public, and many Internets of things are constructed for the purpose of applying this industry, so the benefits are obtained indirectly, and even for the Internet of things system available, its consciousness of demand is not always direct. Therefore, characteristics of its demands are invisible.

For Internet of things, which belongs to invisible demands, it must rely on the end-users' determination to build the system. Nowadays, most industries with the need to use Internet of things have already had mature business operation models, and the introduction of new system is not only a huge investment, but usually represents the establishment of new operation process, especially the Internet of things, a technology meant to collapse the old system. Therefore, it is not likely to persuade enterprise owners to spend a huge amount of money to completely update the old system when it still operates stably, which is why the current development of Internet of things is not as prosperous as what institution and system suppliers have estimated.

\section{Conclusions}

The Internet of things technology is a new thing integrated with multi-disciplinary technologies. Agriculture is an ancient and traditional industry which is regional, seasonal and diversified, which determines that it is complex and difficult to apply the Internet of things technology to traditional agriculture. In developed countries, great development has been made in the connotation of modern agriculture compared with traditional agriculture, and the application of the Internet of things to 
modernized agriculture will play an important role in promoting the modernization of agriculture. With the development of modernized agriculture, application of the Internet of things technology in modern agriculture is gradually expanded. Internet of things is playing a more and more important role and scientific monitoring and cultivation are realized in agricultural products, improving the overall efficiency of agriculture and promoting the transformation and upgrading of modernized agriculture. Promoted by national policies, the industry of the Internet of things is gradually expanding its scale and has already become the most potential industry in the future, and it is also expected to be the most valuable industry in the future. Intelligent agriculture is also one of the industries with great support from the country. More and more people have abandoned the traditional farming pattern and begun to use the sensor to "communicate" with crops, which liberates a considerable amount of human resources and more scientifically and reasonably solve various problems in crops. Thus, it has to be said that this is a progress of the era.

\section{Acknowledgment}

This work was supported by Project on professional and characteristical construction of Jiangxi province 2010 (Civil Engineering) and Project on the planning and construction of disciplines in Jiangxi University of Technology (Structure Engineering)

\section{References}

[1] Zhang Changli, Shen Weizheng. Application of Internet of Things in Agriculture [J]. Journal of Northeast Agricultural University. 2011,05: 1-5.

[2] Jin Pan. Improve Modern Facility Agriculture with Internet of Things [J]. Agricultural Machinery Market. 2010,03: 27-28.

[3] Xu Jianpeng, Zhou Luyang, Zhang Shujing. Application of Internet of Things in Agriculture [J]. Ningxia Agriculture and Forestry Science and Technology. 2012,02: 67-77.

[4] Chen Haiming, Cui Li, Xie Kaibin. Comparative Research of System Structure and Implementation Method of Internet of Things [J]. Journal of Computer Science. 2013,01: 168-188.

[5]Vin Diesel, Collaborative supply chain planning under dynamic lot sizing costs with capacity decision [J]. Journal of Systems Engineering and Electronics, 2011, 02:247-256.

[6] Fan Shanshan, Li Zhong, Chai Rong. Research of the Application of Internet of Things in Intelligent Agriculture [J]. Computer Disc Software and Application. 2013,13: 41-42.

[7] Yan Minjie, Xianing, Wan Zhong, Duan Hongyang. Application of Internet of Things in Modern Agriculture [J]. Chinese Agricultural Science Bulletin. 2011,08: 464-467.

[8] Zheng Hongjian, Yuan Qiuhong, Zhang Lingyun. Research of Key Technologies of Internet of Things Operation Platform and Analysis of Application and Cases [J]. Wireless Technology, 2012, 10: $127-129$.

[9] Qin Huaibin, Li Daoliang, Guo Li. Development of Agricultural Internet of Things and Progress of the Application of Key Technologies [J]. Agricultural Mechanization Research. 2014, 04: 246-252. 
[10] Yu Jianfeng. Development and Practice of Internet of Things Engineering [M]. Beijing: Posts \& Telecom Press. 2013(07).

[11] Chen Haiying, Guo Jiasu, etc. Application Revelation of Internet of Things: Ind ustry Analysis and Case Practice [M]. Beijing: Industrial Publication. 2011(05). 